\title{
AutoMH: Automatically Create Evolutionary Metaheuristic Algorithms Using Reinforced Learning
}

\author{
Boris Almonacid $1,2,+($ C) \\ 1 Global Change Science; boris.almonacid@globalchange.science \\ 2 CoronaWhy. \\ + An extended version is currently in peer review.
}

Received: 31 December 2020

\begin{abstract}
Machine learning research has been able to solve problems in multiple aspects. An open area of research is machine learning for solving optimisation problems. An optimisation problem can be solved using a metaheuristic algorithm, which is able to find a solution in a reasonable amount of time. However, there is a problem, the time required to find an appropriate metaheuristic algorithm, that would have the convenient configurations to solve a set of optimisation problems properly. A solution approach is shown here, using a proposal that automatically creates metaheuristic algorithms aided by a reinforced learning approach. Based on the experiments performed, the approach succeeded in creating a metaheuristic algorithm that managed to solve a large number of different continuous domain optimisation problems. This work's implications are immediate because they describe a basis for the generation of metaheuristic algorithms in real-time.
\end{abstract}

Keywords: Artificial intelligence; Machine Learning; Reinforced Learning; Optimisation; Metaheuristic; Metaheuristic Generation

\section{Introduction}

The use of metaheuristic algorithms has become an approach that is widely used to solve a variety of optimisation problems [1], such as optimisation problems in the field of health, logistics, agriculture, mining, space, robotics, to name a few. The diversity of metaheuristic algorithms in the last decade has grown widely [1], with a great diversity of components, routines, selectors, internals, and especially a great variety of parameters. This diversity leads to different difficulties, such as, for example, being able to find a specific configuration of parameters for a specific type of optimisation problem. A situation that induces and generates a difficulty in being able to choose a metaheuristic algorithm adequately.

Various strategies have been adopted to minimise the effort of manual configurations. One area is machine learning, specifically in reinforced learning [2], where various advances have been made. For example, a general method to reformulate reinforced learning problems as optimisation tasks and apply the particle swarm metaheuristic algorithm to find optimal solutions [3]. A solution to solve the vehicle routing problem [4], Feature Selection [5], a design of a plane frame [6], or Resource Allocation problems [7]. Other approaches include Learnheuristics [8], Q-Learning [9], Meta-learning [10], and Hyper-heuristic [11,12], which provide diverse perspectives on tackling optimisation problems. In [13] the use of Multi-agent reinforcement learning is proposed, which allow for an upgrade in the reinforcement learning area, which generally uses a single agent.

In algorithm generation, there is an approach that uses the construction of a centralised hybrid metaheuristic cooperative strategy to solve optimisation problems [14]. Another approach, [15] uses a set of instructions to create a set of machine learning algorithms in real-time. A basis for understanding the scope of these approaches can be found in [16] which provides the taxonomy of combinations with 
metaheuristics, with mathematical programming, with constraint programming and machine learning. Open problems and a current status of the area can be found in [17], and [18].

For this research, an extension of the basic model of reinforced learning is proposed. The Agent will be called the learning agent, which contains two processes called the analysis process and the action process. The environment is the one that integrates a more significant number of changes because in addition to incorporating a set of optimisation problems to solve, it combines a swarm of non-intelligent agents, which have the purpose of executing the optimisation problems in a metaheuristic algorithm that improves its internal structure during each evolutionary process. The final objective is to be able to find through an evolutionary generation process the best metaheuristic algorithm(s) that solve the set of problems entered by the user.

In the scope of this research, he focuses on contributing within the area of High-level data-driven metaheuristics, specifically on the topic of metaheuristic generation by reinforcement learning. The main benefits expected from this work are as follows:

- Find new metaheuristic algorithms in real-time that solve one or some optimisation problems given by the user.

- Allow the extension of new components to generate new metaheuristic algorithms, as new operators or as new indivisible exploration intensification functions.

- Contribute to the area of machine learning, specifically in the integration of reinforced learning to solve optimisation problems.

The rest of this paper is structured as follow: In section 2, the proposed design and the formalisation of its parts are detailed. In section 3 , the tests performed and their results are detailed. Finally, in section 4 concludes and provides guidelines for future work. 


\section{Materials and Methods}

\subsection{Proposed reinforcement learning framework for the automatic creation of metaheuristics}

The proposed reinforcement learning framework (AutoMH) is to be able to find through a learning agent one or a set of metaheuristic algorithms that are capable of finding the best solution for a portfolio of optimisation problems $P$.

Definition 1. A continuous optimisation problem $P$ is defined by minimise $p(x)$ subject to $l \leq x \leq u$, where $x=\left\{x_{1}, x_{2}, \ldots, x_{d}\right\}, d$ is the dimensions of the optimisation problem, $l=\left\{l_{1}, l_{2}, \ldots, l_{d}\right\}$, and $u=$ $\left\{u_{1}, u_{2}, \ldots, u_{d}\right\}$ are the lower bounds and the upper bounds of the corresponding variables in $x$, which define the feasible domain of the problem.

The figure 1 details the main parts of the framework architecture. It consists of two basic parts of a reinforcement learning system: the Learning Agent and the Environment.

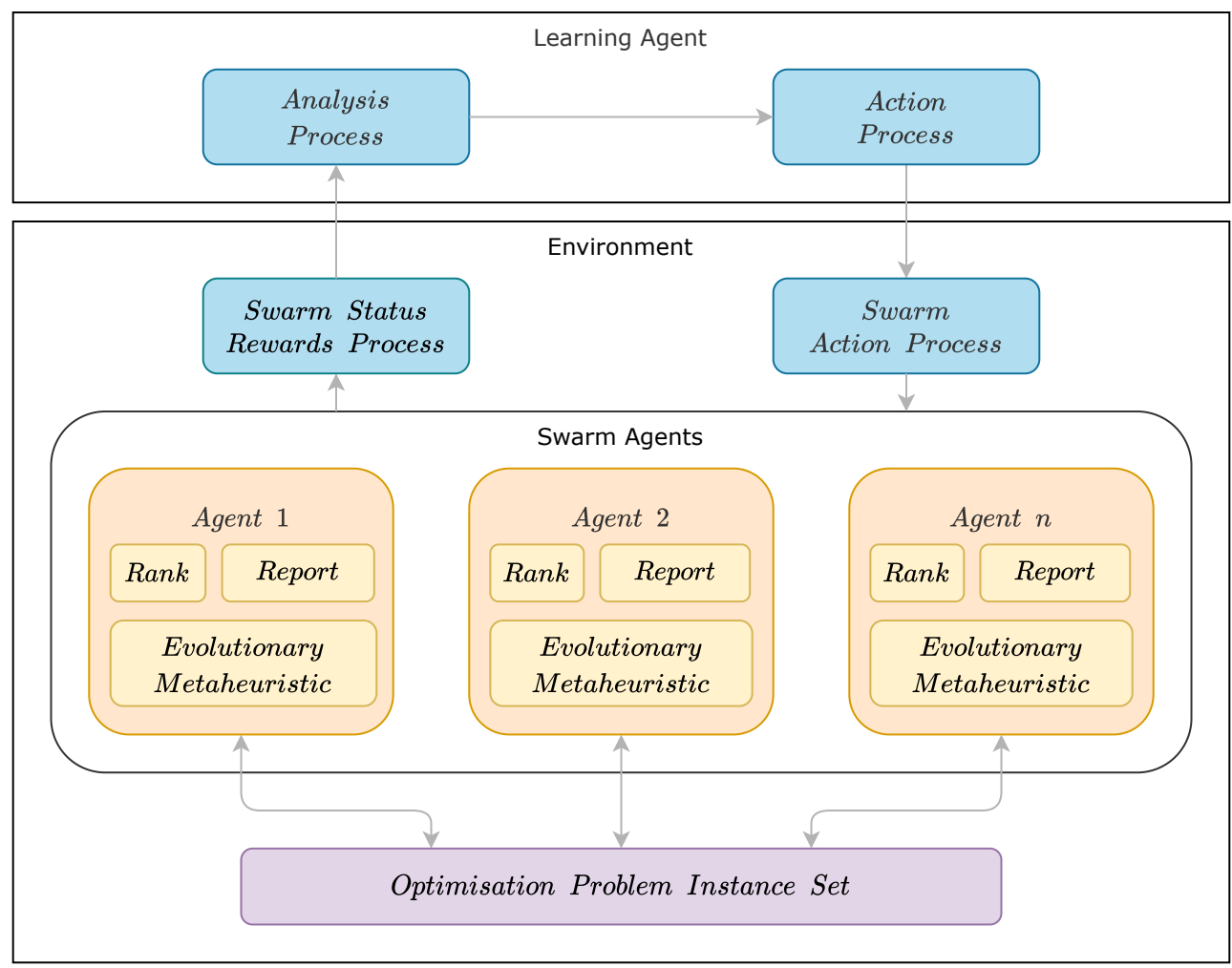

Figure 1. Proposed reinforcement learning framework for the automatic creation of metaheuristics

Learning Agent: The learning agent has the function of analysing the data generated by the environment through the analysis process and taking actions that will affect through a set of actions the internal behaviour of each agent in the swarm of agents through the action process.

Environment: The environment is composed of three elements:

- A Optimisation Problem Instance Set: Corresponds to a portfolio of optimisation problems $P=\left\{p_{1}, p_{2}, \ldots, p_{n}\right\}$ that must be resolved by the agents. An example of optimisation problems is described in the Appendix A, and in the table 5.

- A Swarm Agents: It is a set of non-intelligent agents $A=\left\{A_{1}, A_{2}, \ldots, A_{n}\right\}$.

- Two Swarm Process: The swarm status rewards process to extract information, and the swarm action process to add new information to the agents. 
Swarm Agents: Each agent separately is in charge of executing the tests of the set of optimisation problems $P$. Formally, an agent is determined by Definition 2 .

Definition 2. A Agent $A_{i}$ is defined by the 3-tuple $A_{i}=\langle M, Q, R\rangle$, where:

- A metaheuristic algorithm $M$, which is an empty structure named template. This structure is modified at runtime through the swarm action process.

- A qualification $Q$ corresponds to a variable that indicates the value of the rank assigned to the agent.

- A report $R$ corresponds to a set of data structures in which the results of the optimisation tests are stored. The stored data correspond to a matrix of summaries with best, worst, mean, std, fitness, and solution for each optimisation problem, and a matrix of details of each iteration for each execution of each optimisation problem.

\subsection{Instruction}

An instruction is an ordered grouping of elements with the objective of producing a change in the value of a variable. An instruction is made up of four elements: a variable, an assignment operator, an operator, and a function.

The composition of an instruction is detailed in the equation 1 . Where, from right to left: $f_{x_{t}}$ is the function that is applied using the current value of the variable $x_{t}$ in order to generate a new value, $\Delta$ is the operator that will be applied with the value of the variable $x_{t}$ and with the value obtained by applying the function $f_{x_{t}}$, and the symbol $\leftarrow$ is the assignment operator for a new value that will be assigned in $x_{t+1}$.

$$
\begin{array}{ccccc}
\text { variable } & \text { assignment operator } & \text { variable } & \text { operator } & \text { function } \\
x_{t+1} & \leftarrow & x_{t} & \Delta & f\left(x_{t}\right)
\end{array}
$$

Formally, an instruction is determined by Definition 3. Additionally, instructions can derive into instruction types such as an exploration instruction $\varepsilon$, which is defined by function 2 , or an intensification instruction $\gamma$ that is defined by function 3 .

Definition 3. An instruction I is composed by a variable $x$, one generic instruction operator $O=\left\{\Delta_{k}(x) \mid k \in\right.$ $K\}$, and one exploration function $G=\left\{g_{j}(x) \mid j \in J\right\}$ or one intensification function $H=\left\{h_{i}(x) \mid i \in I\right\}$, where $K=\{1 \ldots l\}, J=\{1 \ldots m\}$, and $I=\{1 \ldots n\}$. The value of $n, m$, and l are determined by the initial information of the system.

$$
\begin{aligned}
& \varepsilon \Leftrightarrow f_{1}\left(x, \Delta_{k}, g_{j}\right)=x \Delta_{k} g_{j}(x) \\
& \gamma \Leftrightarrow f_{2}\left(x, \Delta_{k}, h_{i}\right)=x \Delta_{k} h_{i}(x)
\end{aligned}
$$

For this research, a portfolio of functions with exploration and intensification instructions described in tables 1,2 and 3 will be used. 
Table 1. List of basic functions.

\begin{tabular}{clll}
\hline Identifier & Name & \multicolumn{1}{c}{ Function } & \multicolumn{1}{c}{ Code } \\
\hline I01 & Sine & $f(x)=\sin (x)$ & $\mathrm{x}=\operatorname{SIN}(\mathrm{x})$ \\
I02 & Cosine & $f(x)=\cos (x)$ & $\mathrm{x}=\cos (\mathrm{x})$ \\
I03 & Tangent & $f(x)=\tan (x)$ & $\mathrm{x}=\operatorname{TAN}(\mathrm{x})$ \\
I04 & Inverse Sine & $f(x)=\arcsin (x)$ & $\mathrm{x}=\operatorname{ARCSIN}(\mathrm{x})$ \\
I05 & Inverse Cosine & $f(x)=\arccos (x)$ & $\mathrm{x}=\operatorname{ARCCOS}(\mathrm{x})$ \\
I06 & Inverse Tangent & $f(x)=\arctan (x)$ & $\mathrm{x}=\operatorname{ARCTAN}(\mathrm{x})$ \\
I07 & Absolute & $f(x)=|x|$ & $\mathrm{x}=\operatorname{ABS}(\mathrm{X})$ \\
I08 & Square root & $f(x)=\sqrt{x}$ & $\mathrm{x}=\operatorname{SQRT}(\mathrm{x})$ \\
I09 & Exponential function & $f(x)=e^{x}$ & $\mathrm{x}=\operatorname{EXP}(\mathrm{X})$ \\
I10 & Exponential function minus 1 & $f(x)=e^{x}-1$ & $\mathrm{x}=\operatorname{EXP1}(\mathrm{x})$ \\
I11 & Natural logarithm & $f(x)=\ln (x)$ & $\mathrm{x}=\operatorname{LN}(\mathrm{x})$ \\
I12 & Base-2 logarithm of $\mathrm{x}$ & $f(x)=\log _{2}(x)$ & $\mathrm{x}=\operatorname{LOG} 2(\mathrm{x})$ \\
I13 & Base-10 logarithm of $\mathrm{x}$ & $f(x)=\log _{10}(x)$ & $\mathrm{x}=\operatorname{LOG10}(\mathrm{x})$ \\
I14 & natural logarithm of one plus & $f(x)=\ln (1+x)$ & $\mathrm{x}=\operatorname{LN}(1+\mathrm{X})$ \\
\hline
\end{tabular}

Table 2. List of random number functions.

\begin{tabular}{|c|c|c|c|c|}
\hline Identifier & Name & Function & Code & Description \\
\hline I100 & Uniform F1 & $U_{f 1} \sim(l, u)$ & $\mathrm{x}=\operatorname{UNIFORM} 1(\mathrm{~L}, \mathrm{U})$ & \\
\hline I101 & Uniform F2 & $U_{f 2} \sim(l, u)$ & $\mathrm{x}=\mathrm{UNIFORM} 2(\mathrm{~L}, \mathrm{U})$ & $\mathrm{u}=\mathrm{lb}+(\mathrm{ub}-\mathrm{lb}) / 2$ \\
\hline I102 & Uniform F3 & $U_{f 3} \sim(l, u)$ & $\mathrm{x}=\operatorname{UNIFORM} 3(\mathrm{~L}, \mathrm{U})$ & $1=\mathrm{lb}+(\mathrm{ub}-\mathrm{lb}) / 2$ \\
\hline I103 & Uniform F4 & $U_{f 4} \sim(l, u)$ & $\mathrm{x}=\operatorname{UNIFORM} 4(\mathrm{~L}, \mathrm{U})$ & $\begin{array}{l}1=\mathrm{lb}+(\mathrm{ub}-\mathrm{lb}) / 3 \\
\mathrm{u}=\mathrm{lb}+(\mathrm{ub}-\mathrm{lb}) / 3^{*} 2\end{array}$ \\
\hline I104 & Uniform F5 & $U_{f 5} \sim(l, u)$ & $\mathrm{x}=\operatorname{UNIFORM} 5(\mathrm{~L}, \mathrm{U})$ & $\mathrm{u}=\mathrm{lb}+(\mathrm{ub}-\mathrm{lb}) / 4$ \\
\hline I105 & Uniform F6 & $U_{f 6} \sim(l, u)$ & $\mathrm{x}=\operatorname{UNIFORM} 6(\mathrm{~L}, \mathrm{U})$ & $\begin{array}{l}\mathrm{l}=\mathrm{lb}+(\mathrm{ub}-\mathrm{lb}) / 4 \\
\mathrm{u}=\mathrm{lb}+(\mathrm{ub}-\mathrm{lb}) / 2\end{array}$ \\
\hline I106 & Uniform F7 & $U_{f 7} \sim(l, u)$ & $\mathrm{x}=\operatorname{UNIFORM7}(\mathrm{L}, \mathrm{U})$ & $\begin{array}{l}\mathrm{l}=\mathrm{lb}+(\mathrm{ub}-\mathrm{lb}) / 2 \\
\mathrm{u}=\mathrm{lb}+(\mathrm{ub}-\mathrm{lb}) / 4^{* 3}\end{array}$ \\
\hline I107 & Uniform F8 & $U_{f 8} \sim(l, u)$ & $\mathrm{x}=\mathrm{UNIFORM} 8(\mathrm{~L}, \mathrm{U})$ & $1=\mathrm{lb}+(\mathrm{ub}-\mathrm{lb}) / 4^{*} 3$ \\
\hline I108 & Uniform F9 & $U_{f 9} \sim(-1,1)$ & $\mathrm{x}=\operatorname{UNIFORM} 9(-1,1)$ & \\
\hline I109 & Uniform F10 & $U_{f 10} \sim(0,1)$ & $\mathrm{x}=\operatorname{UNIFORM} 10(0,1)$ & \\
\hline I110 & Uniform F11 & $U_{f 11} \sim(-1,0)$ & $\mathrm{x}=\operatorname{UNIFORM} 11(-1,0)$ & \\
\hline I111 & Uniform F12 & $U_{f 12} \sim(0.5,0.5)$ & $\mathrm{x}=$ UNIFORM12(0.5, 0.5) & \\
\hline I112 & Beta F1 & $B_{f 1} \sim(0.5,0.5,1)$ & $x=\operatorname{BETA} 1(0.5,0.5,1)$ & \\
\hline I113 & Beta F2 & $B_{f 2} \sim(5,1,1)$ & $x=\operatorname{BETA} 2(5,1,1)$ & \\
\hline I114 & Beta F3 & $B_{f 3} \sim(1,3,1)$ & $x=\operatorname{BETA} 3(1,3,1)$ & \\
\hline I115 & Beta F4 & $B_{f 4} \sim(2,2,1)$ & $x=\operatorname{BETA} 4(2,2,1)$ & \\
\hline I116 & Beta F5 & $B_{f 5} \sim(2,5,1)$ & $x=\operatorname{BETA} 5(2,5,1)$ & \\
\hline I117 & Triangular F1 & $T_{f 1} \sim(l b, m, u b)$ & $\mathrm{x}=$ TRIANGULAR1 $(\mathrm{LB}, \mathrm{M}, \mathrm{UB})$ & $\mathrm{m}=\mathrm{lb}+(\mathrm{ub}-\mathrm{lb}) / 2$ \\
\hline I118 & Triangular F2 & $T_{f 2} \sim(l b, m, u b)$ & $\mathrm{x}=$ TRIANGULAR2 $(\mathrm{LB}, \mathrm{M}, \mathrm{UB})$ & $\mathrm{m}=\mathrm{lb}+(\mathrm{ub}-\mathrm{lb}) / 4$ \\
\hline I119 & Triangular F3 & $T_{f 3} \sim(l b, m, u b)$ & $\mathrm{x}=$ TRIANGULAR3(LB, M, UB) & $\mathrm{m}=\mathrm{lb}+(\mathrm{ub}-\mathrm{lb}) / 3$ \\
\hline I120 & Triangular F4 & $T_{f 4} \sim(l b, m, u b)$ & $\mathrm{x}=$ TRIANGULAR4 $(\mathrm{LB}, \mathrm{M}, \mathrm{UB})$ & $\mathrm{m}=\mathrm{lb}+((\mathrm{ub}-\mathrm{lb}) / 4)^{* 3}$ \\
\hline I121 & Triangular F5 & $T_{f 5} \sim(l b, m, u b)$ & $\mathrm{x}=$ TRIANGULAR5 $(\mathrm{LB}, \mathrm{M}, \mathrm{UB})$ & $\mathrm{m}=\mathrm{lb}+((\mathrm{ub}-\mathrm{lb}) / 3)^{* 2}$ \\
\hline
\end{tabular}


Table 3. List of constants.

\begin{tabular}{clcll}
\hline ID & Name & Symbol & Value & Code \\
\hline I200 & Meissel-Mertens & $M_{1}$ & 0.26149721284764278375542683860869585 & A077761( ) \\
I201 & Bernstein's & $\beta$ & 0.28016949902386913303 & A073001( ) \\
I202 & Gauss-Kuzmin-Wirsing & $\lambda$ & 0.30366300289873265859744812190155623 & A038517( ) \\
I203 & Hafner-Sarnak-McCurley & $\sigma$ & 0.35323637185499598454351655043268201 & A085849( ) \\
I204 & Omega & $\Omega$ & 0.56714329040978387299996866221035554 & A030178( ) \\
I205 & Euler-Mascheroni & $\gamma$ & 0.57721566490153286060651209008240243 & A001620( ) \\
I206 & Twin prime & $C_{2}$ & 0.66016181584686957392781211001455577 & A005597( ) \\
I207 & Conway's & $\lambda_{c}$ & 1.30357726903429639125709911215255189 & A014715( ) \\
I208 & Ramanujan-Soldner & $\mu$ & 1.45136923488338105028396848589202744 & A070769( ) \\
I209 & Golden ratio & $\varphi$ & 1.61803398874989484820458683436563811 & A001622( ) \\
I210 & Euler's number & $e$ & 2.71828182845904523536028747135266249 & A001113( ) \\
I211 & Pi & $\pi$ & 3.14159265358979323846264338327950288 & A000796( ) \\
I212 & Reciprocal Fibonacci & $\psi$ & 3.35988566624317755317201130291892717 & A079586( ) \\
\hline
\end{tabular}

Definition 4. An operator $\Delta$ is a mathematical symbol that indicates that a specific operation must be performed on a variable and a function.

Table 4. List of operators.

\begin{tabular}{clll}
\hline Identifier & Name & \multicolumn{1}{c}{ Math } & \multicolumn{1}{c}{ Code } \\
\hline O00 & None & $x \leftarrow x$ & $\mathrm{x}=\mathrm{x}$ \\
O01 & Plus & $x \leftarrow x+f(x)$ & $\mathrm{x}=\mathrm{x}+\mathrm{f}(\mathrm{x})$ \\
O02 & Subtract & $x \leftarrow x-f(x)$ & $\mathrm{x}=\mathrm{x}-\mathrm{f}(\mathrm{x})$ \\
O03 & Multiply & $x \leftarrow x * f(x)$ & $\mathrm{x}=\mathrm{x}$ f $(\mathrm{x})$ \\
O04 & Divide & $x \leftarrow \frac{x}{f(x)}$ & $\mathrm{x}=\mathrm{x} / \mathrm{f}(\mathrm{x})$ \\
\hline
\end{tabular}

\subsection{Evolutionary metaheuristic algorithm}

A metaheuristic algorithm $M$ is a template that changes in each cycle depending on the decisions made by the learning agent through the action process. Changes to its structure are made at runtime through the swarm action process. The structure of the metaheuristic algorithm template is constituted by the SETUP, STEP, and END functions.

Definition 5. An evolutionary metaheuristic algorithm is defined by the 3-tuple $M=\langle\tau, E, \Gamma\rangle$, where: $\tau$ is a metaheuristic template that is composed by SETUP, STEP, END, and RUN functions, $E$ is a dynamic array of exploration functions $E=\left[\epsilon_{1}, \epsilon_{2}, \ldots, \epsilon_{n}\right]$, and $\Gamma$ is a set of intensification functions $\Gamma=\left[\gamma_{1}, \gamma_{2}, \ldots, \gamma_{n}\right]$.

The description of the functions are described below:

- The STARTED function is in charge of initialising the variables of the optimisation problem. Initialisation is carried out using one or more scan instructions. Subsequently, the current fitness is calculated and the solution is stored.

- The STEP function is the main core of the template. In this function the main modifications are made in the evolutionary metaheuristic algorithm. Actions are carried out such as adding, modifying or deleting instructions both of the type of exploration instructions, as well as intensification instructions. Subsequently, the new aptitude of the solution is calculated, and the new aptitude and solution is stored in the event that it is better than the previous one.

- The END function is executed when the end condition of the metaheuristic algorithm ends. Its function is to extract the solution found and its associated aptitude.

Figure 2 describes an example of a template that has already been modified by the learning agent. The STARTED function has a single instruction that is composed of the operator NONE with the code 
O00, and by the UNIFORM10 $(0,1)$ function with the code I109. The STEP function is composed of a exploration instruction $<\mathrm{O} 02$, I06 $>$ and two intensification instructions $<\mathrm{OO} 2, \mathrm{I} 06>$ and $<\mathrm{O} 03, \mathrm{I} 14>$.

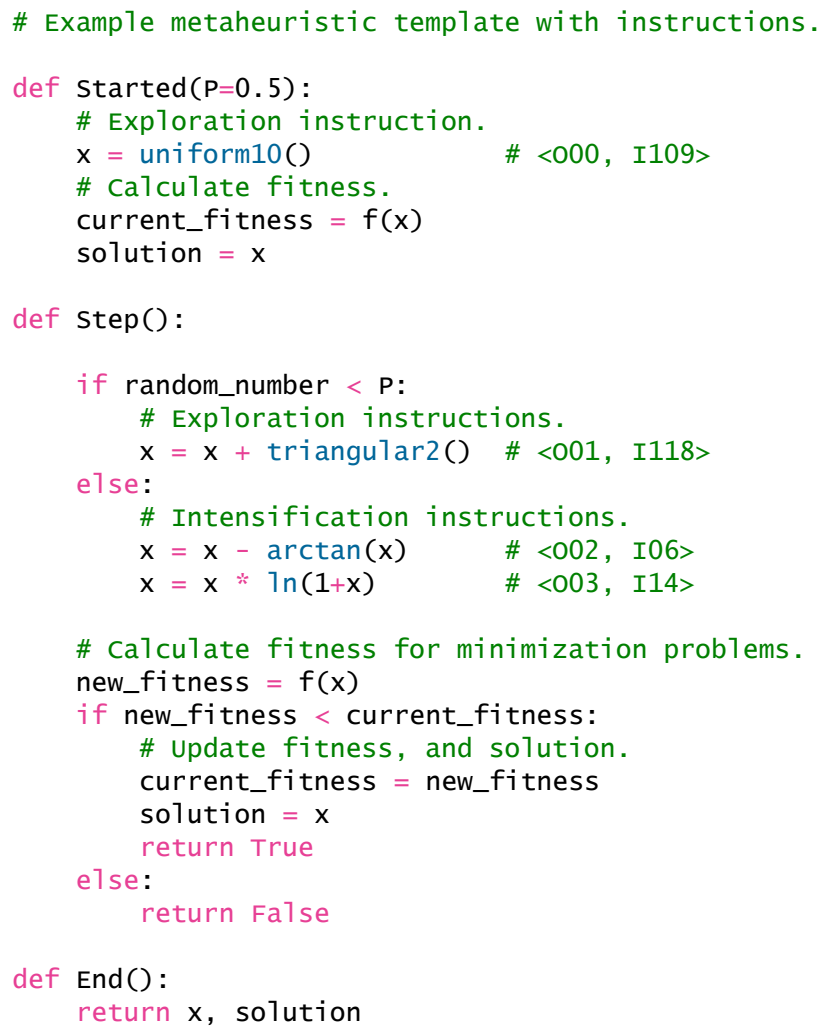

Figure 2. Metaheuristic Template

The Run function has the function of executing the STARTED, STEP and END functions. From executing the Started function to initialise the algorithm, executing the while loop with the calls to the STEP function, and ending the algorithm when the stopping criterion is met. When this condition is fulfilled, the End function will be called to extract the final solution of the optimisation problem $P$. The pseudocode of the RUN function is described in figure 3.

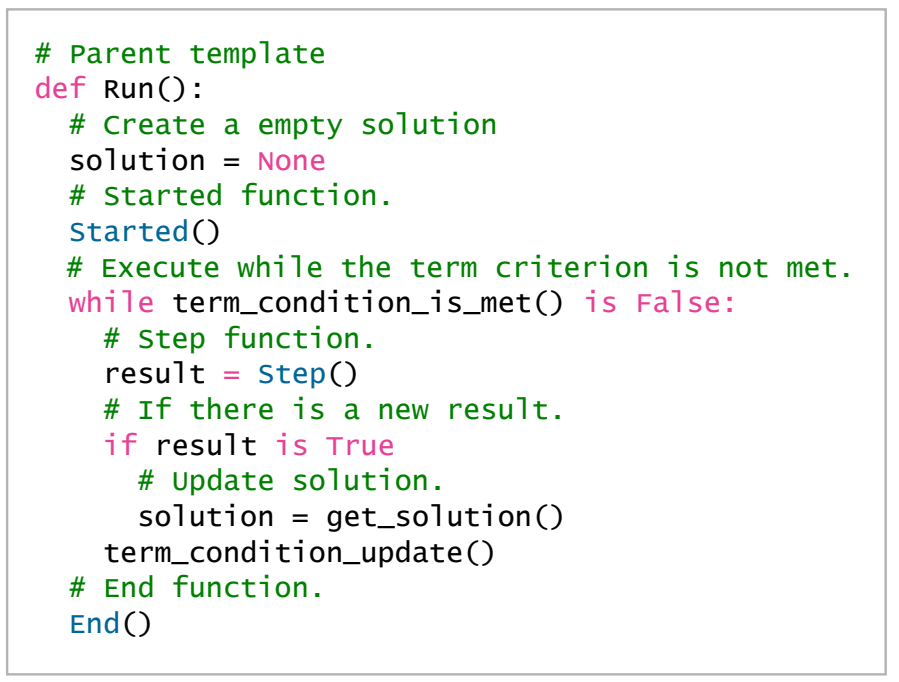

Figure 3. Parent Template 


\subsection{Swarm Status Rewards Process}

The Swarm Status Rewards Process consists of a process to collect the information generated by the swarm of agents when executing the metaheuristic algorithm. Store the information for each problem $P$, for each execution of the problem $P$, the results regarding the best solution, the fitness, and the fitness values for each iteration. The values are stored in a structure called Report. Report $=\{$ best_solutions, best_fitness, fitness_iterations $\}$.

\subsection{Analysis Process}

The objective is to rank each agent in the swarm. The measure used to order the agents is the average value obtained for each optimisation problem. The average value is represented by the matrix $Q \in \mathbb{R}^{m \times n}$ (See 4), where: $m \in\{1, p\} n \in\{1, a\}, p$ is the number of optimisation problems and $a$ is the number of agents in the swarm.

$$
Q_{m, n}=\left[\begin{array}{cccc}
q_{1,1} & q_{1,2} & \cdots & q_{1, n} \\
q_{2,1} & q_{2,2} & \cdots & q_{2, n} \\
\vdots & \vdots & \ddots & \vdots \\
q_{m, 1} & q_{m, 2} & \cdots & q_{m, n}
\end{array}\right]
$$

Subsequently, a series of operations are carried out:

1. The assignment of ranges is carried out using the data provided by the $Q$ matrix. The method used is the minimum (competition) method, which consists in that to perform the ranking to each value, the minimum of the ranges that would have been assigned is assigned to all tied values. The ranking result is stored in matrix $R \in \mathbb{N}^{m \times n}$ (See 5), where: $m \in\{1, p\} n \in\{1, a\}, p$ is the number of optimisation problems and $a$ is the number of agents in the swarm. The minimum method is performed for each row of the matrix $Q$, which will consider that each problem will have its own ranking among all the agents. The ranking result for each row will be stored in matrix $R$. (See 6), where: $n \in\{1, a\}$, and $a$ is the number of agents in the swarm.

$$
R_{m, n}=\left[\begin{array}{cccc}
r_{1,1} & r_{1,2} & \cdots & r_{1, n} \\
r_{2,1} & r_{2,2} & \cdots & r_{2, n} \\
\vdots & \vdots & \ddots & \vdots \\
r_{m, 1} & r_{m, 2} & \cdots & r_{m, n}
\end{array}\right]
$$

2. A sum is made by the value of each column in matrix $R$. Each sum will correspond to the final ranking value for each agent in the swarm. The values of each sum are stored in a vector $S \in \mathbb{N}^{n}$

$$
S_{n}=\left[\begin{array}{llll}
\sum_{i=1}^{m} r_{i, 1} & \sum_{i=1}^{m} r_{i, 2} & \cdots & \sum_{i=1}^{m} r_{i, n}
\end{array}\right]
$$

\subsection{Action Process}

The action process takes the information generated by the analysis process and performs a swarm modification procedure. The steps performed are:

1. Sort the swarm agents from the best ranking to the worst ranking.

2. Count in a variable $c$ the number of agents with the best ranking.

3. Eliminate an amount $c$ of the worst agents of the swarm.

4. Make a copy of the best agents in an amount $c$ and add them to the end of the swarm.

5. Mark the best agents in an amount $c$ with the status NONE.

6. Mark the rest of the agents with the status MODIFY. 


\section{Experiments}

This section describes the characteristics of the tests and the results obtained by their execution.

Methodology: The methodology consists of collecting a portfolio of continuous function optimisation problems. Perform configuration learning system with a fixed number of agents, evolutionary iterations to be performed, and the type of mutation. For each non-intelligent agent, including the number of iterations and the number of executions each metaheuristic algorithm will perform. Finally, incorporate a list of intensification functions, exploration functions, and operators that will be used to create the instructions. Table 5 indicates the parameters used in the experiment.

Environment: The experiment was developed in Python language version 3.8.5, running on a MSI P65 Creator 9SE laptop with Intel Core I7 9750H CPUs @ 2.60Ghz, 16 GB RAM, and Windows 10 Pro Os build 19041.685.

Table 5. Parameters used in the tests.

\begin{tabular}{|c|c|c|}
\hline Name & Value & Description \\
\hline Number of agents & 30 & The number of agents in the swarm. \\
\hline Evolutionary iterations & 200 & $\begin{array}{l}\text { The number of times the agents in the } \\
\text { swarm have to repeat the optimisation tests } \\
\text { once the structure of their algorithm is } \\
\text { modified by the learning agent. }\end{array}$ \\
\hline Mutation Selection & Random & $\begin{array}{l}\text { The mutation is carried out by randomly } \\
\text { choosing an action of the type }\{A D D \\
\text { REPLACE, REMOVE }\} \text {. }\end{array}$ \\
\hline Metaheuristic iterations & 100 & $\begin{array}{l}\text { The maximum number of iterations that the } \\
\text { metaheuristic executes. }\end{array}$ \\
\hline Metaheuristic executions & 5 & $\begin{array}{l}\text { The number of times the metaheuristic is } \\
\text { executed. }\end{array}$ \\
\hline Operators & $\mathrm{O} 01, \mathrm{O} 02, \mathrm{O} 03, \mathrm{O} 04$ & $\begin{array}{l}\text { The operators allowed to modify the } \\
\text { metaheuristic template. }\end{array}$ \\
\hline Started & I100, I103, I108, I109, I110, I111 & $\begin{array}{l}\text { The exploration functions allowed for } \\
\text { modifying the metaheuristic template in the } \\
\text { STARTED function. }\end{array}$ \\
\hline Intensification & $\begin{array}{l}\text { I01, I02, I03, I04, I05, I06, I07, I08, } \\
\text { I09, I10, I11, I12, I13, I14 }\end{array}$ & $\begin{array}{l}\text { The intensification functions allowed for the } \\
\text { modification of the metaheuristic template } \\
\text { in the STEP function. }\end{array}$ \\
\hline Exploration & $\begin{array}{l}\text { I100, I101, I102, I103, I104, I105, } \\
\text { I106, I107, I108, I109, I110, I111, } \\
\text { I112, I113, I114, I115, I116, I117, } \\
\text { I118, I119, I120, I121, I200, I201, } \\
\text { I202, I203, I204, I205, I206, I207, } \\
\text { I208, I209, I210, I211, I212 }\end{array}$ & $\begin{array}{l}\text { The exploration instructions allowed for the } \\
\text { modification of the metaheuristic template } \\
\text { in the STEP function. }\end{array}$ \\
\hline Dimension & 20 & $\begin{array}{l}\text { The dimension of the optimisation } \\
\text { problems. }\end{array}$ \\
\hline
\end{tabular}

Description of the dataset: Table 6 describes 13 types of problems collected in the literature. Problems P01 to P07 correspond to unimodal functions, and problems P08 to P13 correspond to multimodal functions. Detailed descriptions of these functions are given in Appendix A. 
Table 6. Continuous optimisation problem dataset

\begin{tabular}{|c|c|c|c|c|c|}
\hline Identifier & Function Name & Domain & $f_{\min }\left(x^{*}\right)$ & $x^{*}=\left[x_{1}, x_{2}, \ldots, x_{n}\right]$ & Details \\
\hline P01 & Sphere & {$[-100,100]$} & 0 & $f(0,0, \ldots, 0)$ & A1 \\
\hline P02 & Schwefel Function 2.22 & {$[-10,10]$} & 0 & $\mathrm{f}(0,0, \ldots, 0)$ & $\mathrm{A} 2$ \\
\hline P03 & Schwefel Function 1.2 & {$[-100,100]$} & 0 & $\mathrm{f}(0,0, \ldots, 0)$ & A3 \\
\hline P04 & Schwefel Function 2.21 & {$[-100,100]$} & 0 & $\mathrm{f}(0,0, \ldots, 0)$ & A4 \\
\hline P05 & Rosenbrock's & {$[-30,30]$} & 0 & $\mathrm{f}(1,1, \ldots, 1)$ & A5 \\
\hline P06 & Step & {$[-100,100]$} & 0 & $\begin{array}{c}\mathrm{f}\left(x_{1}, x_{2}, \ldots, x_{d}\right) \\
x_{i} \in[-0.5,0.5), i=\{1,2, \ldots, d\}\end{array}$ & A6 \\
\hline P07 & Quartic & {$[-1.28,1.28]$} & 0 & $f(0,0, \ldots, 0)$ & A7 \\
\hline P08 & Schwefel Function 2.26 & {$[-500,500]$} & 0 & $\begin{array}{c}\mathrm{f}(4.209687462275036 \mathrm{e} 2, \ldots, \\
4.209687462275036 \mathrm{e} 2)\end{array}$ & A8 \\
\hline P09 & Rastrigin & {$[-5.12,5.12]$} & 0 & $\mathrm{f}(0,0, \ldots, 0)$ & A9 \\
\hline P10 & Ackley & {$[-32,32]$} & 0 & $\mathrm{f}(0,0, \ldots, 0)$ & A10 \\
\hline P11 & Griewank & {$[-600,600]$} & 0 & $\mathrm{f}(0,0, \ldots, 0)$ & A11 \\
\hline P12 & $\begin{array}{l}\text { Generalized Penalized } \\
\text { Function } 1\end{array}$ & {$[-50,50]$} & 0 & $\mathrm{f}(1,1, \ldots, 1)$ & A12 \\
\hline P13 & $\begin{array}{l}\text { Generalized Penalized } \\
\text { Function } 2\end{array}$ & {$[-50,50]$} & 0 & $\mathrm{f}(1,1, \ldots, 1)$ & A13 \\
\hline
\end{tabular}

\subsection{Results}

Table 7 describes the results obtained at the end of the test with the 200 evolutionary iterations. This result corresponds to the best agent in the swarm. The results are the best, and worst fitness found for 13 continuous optimisation problems. In addition, the average and standard deviation of the fitness have been incorporated.

Table 7. The description of the table has the following attributes: Column 1 (Identifier) indicates the assigned identifier for each test instance. Column 2 (Best) indicate the best optimum value found. Column 3 (Mean), and column 4 (Std.) describe the mean, and standard deviation respectively Column 5 (Worst) indicate the worst optimum value found.

\begin{tabular}{crrrr}
\hline Identifier & \multicolumn{1}{c}{ Best } & \multicolumn{1}{c}{ Mean } & \multicolumn{1}{c}{ Std. } & \multicolumn{1}{c}{ Worst } \\
\hline P1 & 0.0000 & 0.0000 & 0.0000 & 0.0000 \\
P2 & 0.0000 & 0.0000 & 0.0000 & 0.0000 \\
P3 & 0.0000 & 0.0000 & 0.0000 & 0.0000 \\
P4 & 0.0000 & 0.0000 & 0.0000 & 0.0000 \\
P5 & 18.8171 & 18.8171 & 0.0000 & 18.8171 \\
P6 & 0.0000 & 0.0000 & 0.0000 & 0.0000 \\
P7 & 0.0000 & 0.0000 & 0.0000 & 0.0000 \\
P8 & 4767.8746 & 4767.8746 & 0.0000 & 4767.8746 \\
P9 & 0.0000 & 0.0000 & 0.0000 & 0.0000 \\
P10 & 0.0000 & 0.0000 & 0.0000 & 0.0000 \\
P11 & 0.0000 & 0.0000 & 0.0000 & 0.0000 \\
P12 & 0.1167 & 0.2373 & 0.0749 & 0.3375 \\
P13 & 1.0750 & 1.0750 & 0.0000 & 1.0750 \\
\hline
\end{tabular}

The instructions with the best algorithm found during the 200 evolutionary iterations are:

Started function: Instruction for generating random solution.

1. $<\mathrm{OO} 00, \mathrm{I} 111>$

Step function: Intensification instructions.

1. $<\mathrm{O} 04, \mathrm{I} 01>$

2. $<\mathrm{OO} 3, \mathrm{I} 12>$ 
3. $<\mathrm{OO} 1, \mathrm{I} 10>$

Step function: Exploration instructions.

1. $<\mathrm{O} 04, \mathrm{I} 202>$

2. $<\mathrm{O} 04, \mathrm{I} 200>$

3. $<\mathrm{OO} 3, \mathrm{I} 117>$

4. $<\mathrm{O} 02, \mathrm{I} 109>$

5. $<\mathrm{O} 02, \mathrm{I} 200>$

Figures 5, 6, 7, 8, 9, 10, 11, 12, 13, 14, 15, 16, and 17 show the convergence of the evolutionary algorithm during all iterations for each execution performed. Additionally, it has built an enlarged view of convergence when the metaheuristic find the best fitness.
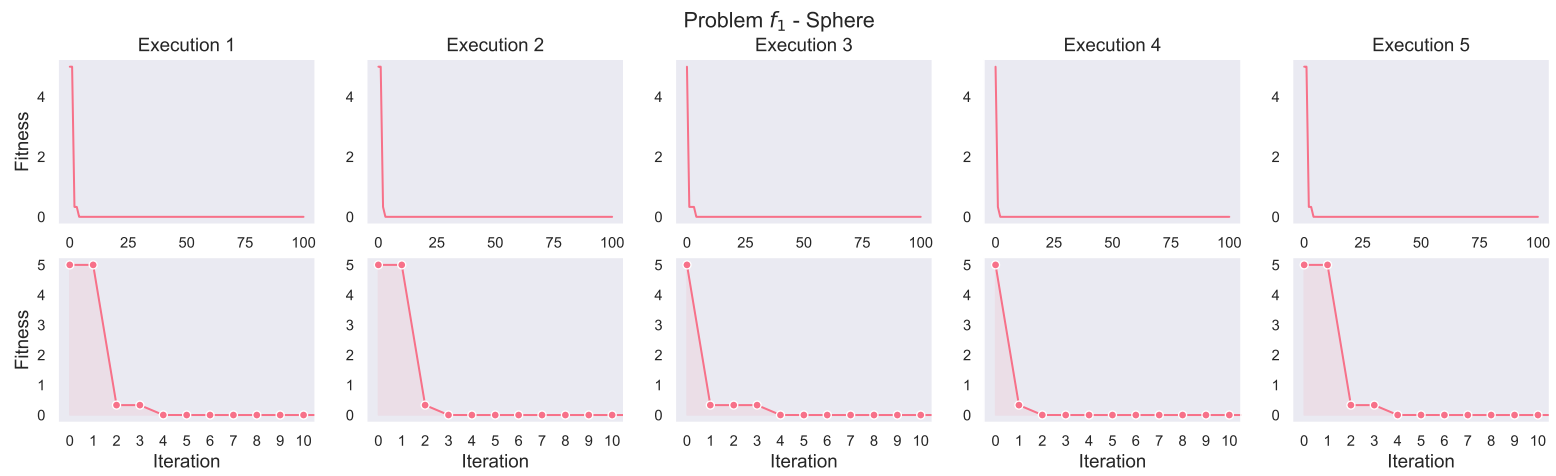

Figure 5. Convergence graph for five executions of problem F1 - Sphere. The top graph describes the convergence from iteration 0 to iteration 100. The lower graph describes the moment in which the best aptitude is obtained during the execution of the algorithm.

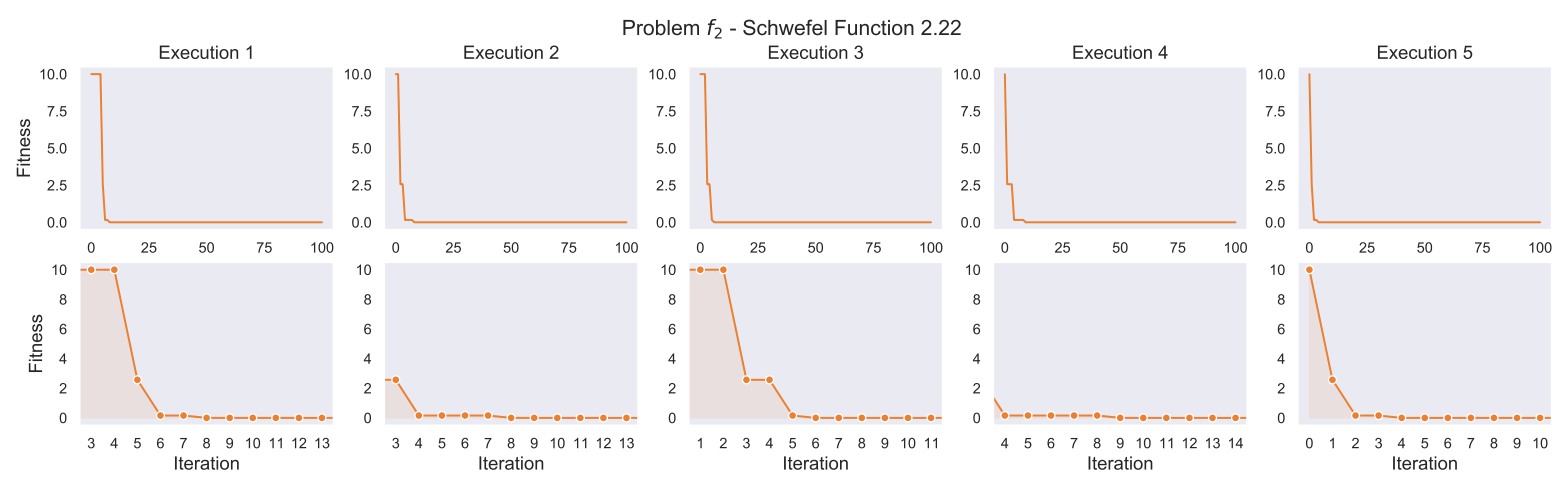

Figure 6. Convergence graph for five executions of problem F2 - Schwefel Function 2.22. The top graph describes the convergence from iteration 0 to iteration 100. The lower graph describes the moment in which the best aptitude is obtained during the execution of the algorithm. 

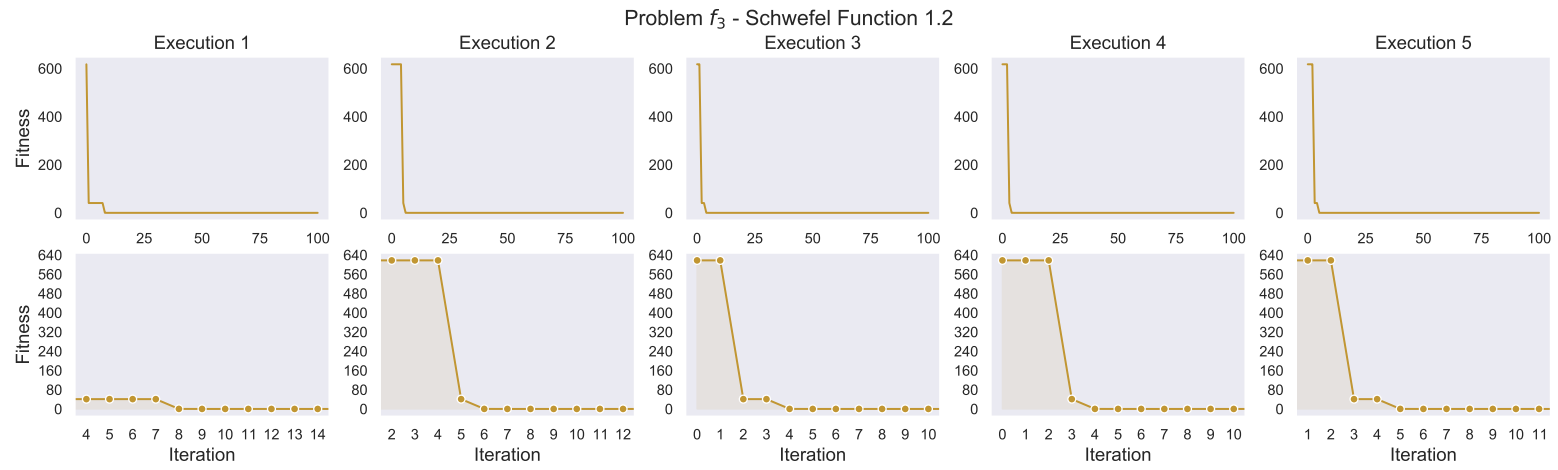

Figure 7. Convergence graph for five executions of problem F3 - Schwefel Function 1.2. The top graph describes the convergence from iteration 0 to iteration 100. The lower graph describes the moment in which the best aptitude is obtained during the execution of the algorithm.
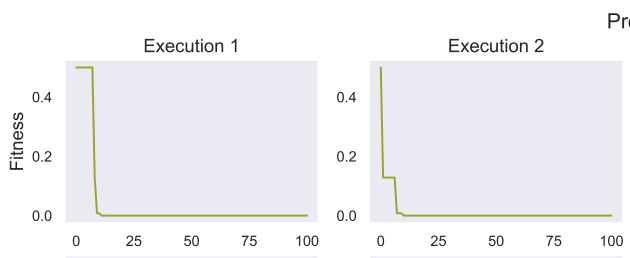

Problem $f_{4}$ - Schwefel Function 2.21
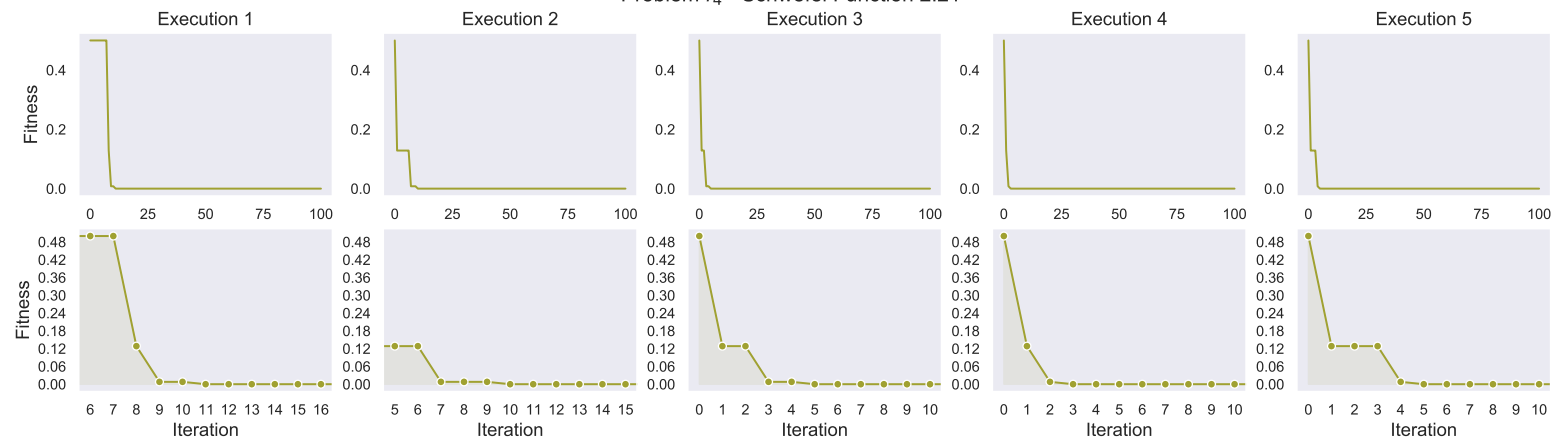

Figure 8. Convergence graph for five executions of problem F4 - Schwefel Function 2.21. The top graph describes the convergence from iteration 0 to iteration 100. The lower graph describes the moment in which the best aptitude is obtained during the execution of the algorithm.
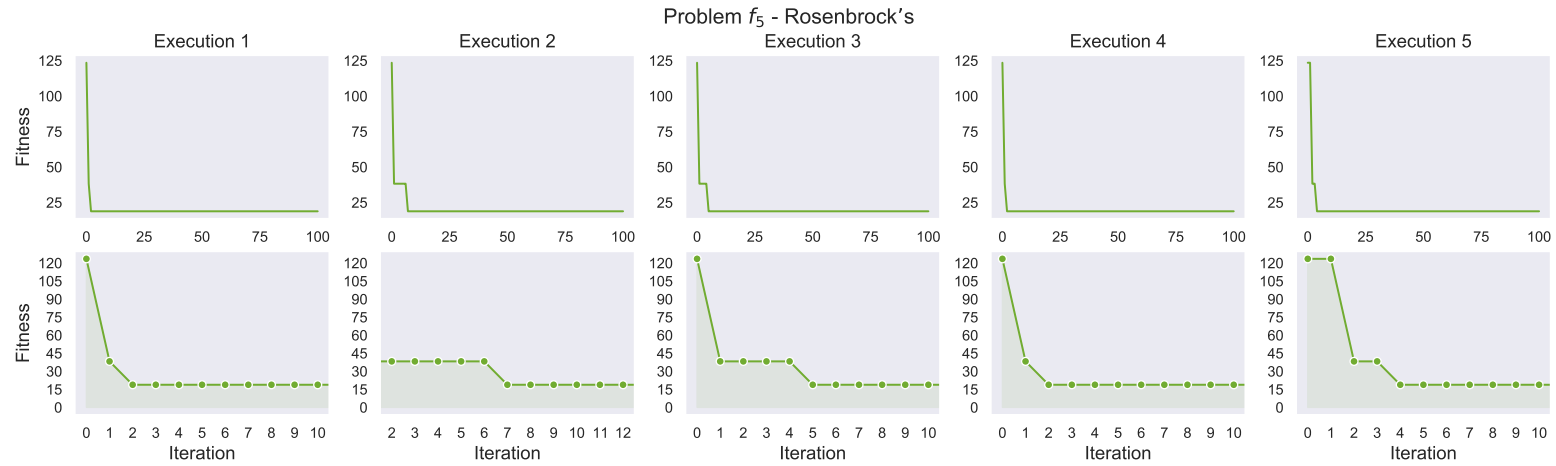

Figure 9. Convergence graph for five executions of problem F5 - Rosenbrock's. The top graph describes the convergence from iteration 0 to iteration 100. The lower graph describes the moment in which the best aptitude is obtained during the execution of the algorithm. 

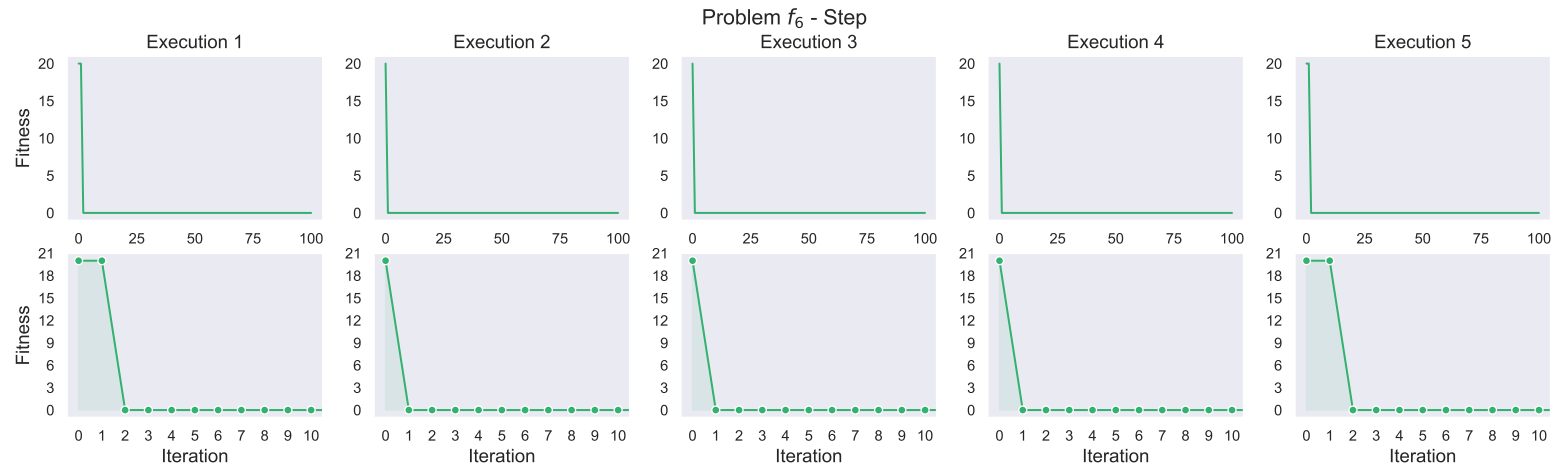

Figure 10. Convergence graph for five executions of problem F6 - Step. The top graph describes the convergence from iteration 0 to iteration 100. The lower graph describes the moment in which the best aptitude is obtained during the execution of the algorithm.
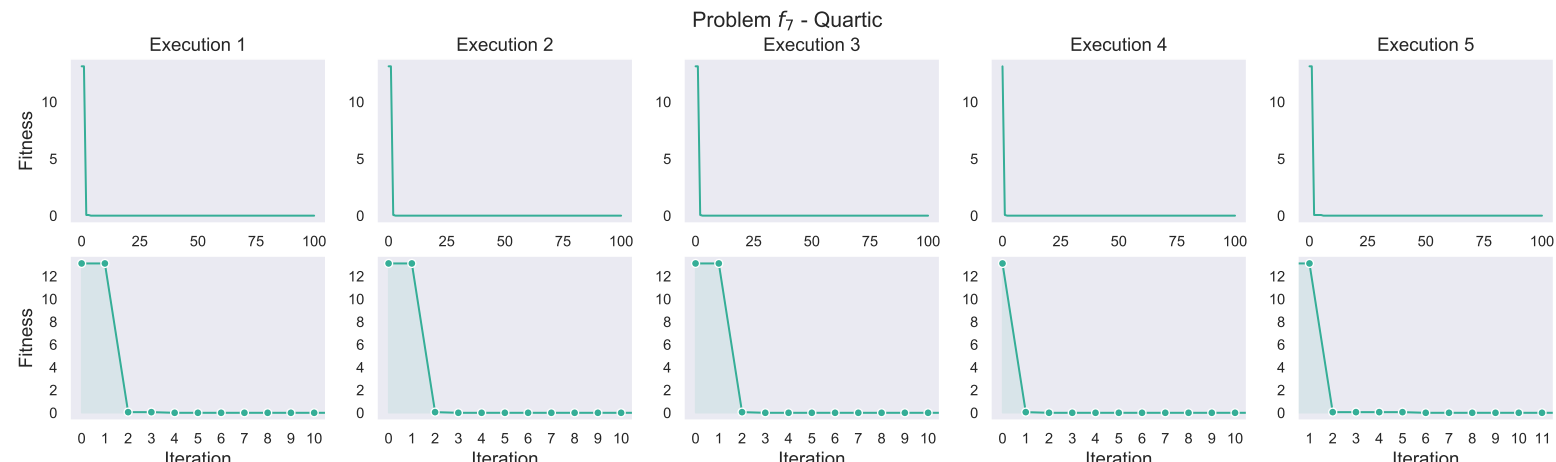

Figure 11. Convergence graph for five executions of problem F7 - Quartic. The top graph describes the convergence from iteration 0 to iteration 100. The lower graph describes the moment in which the best aptitude is obtained during the execution of the algorithm.
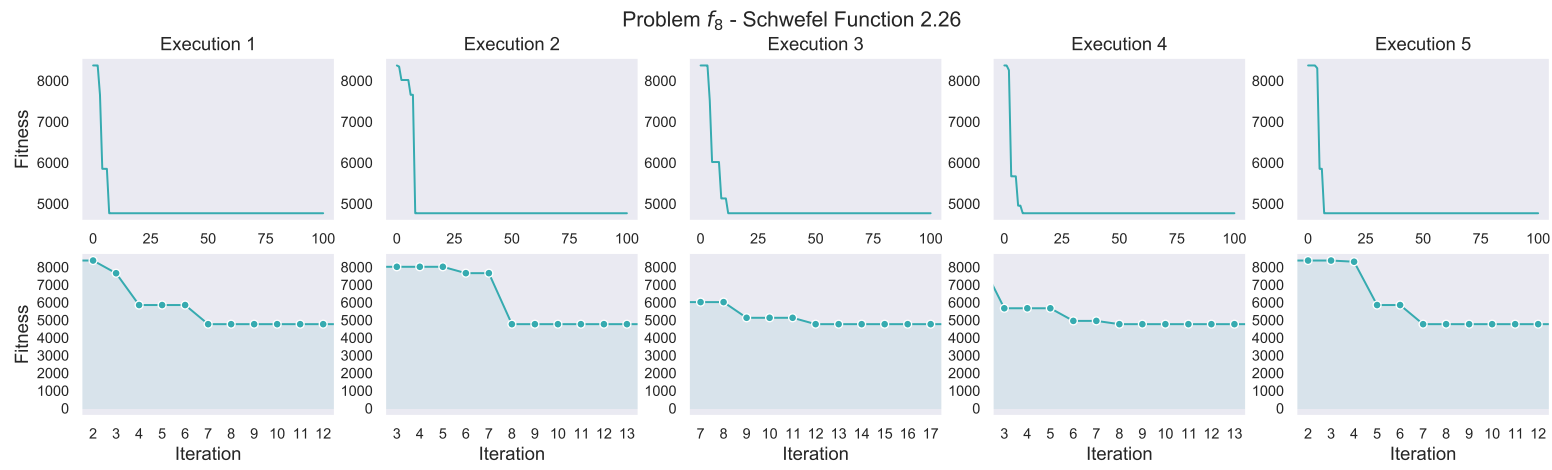

Figure 12. Convergence graph for five executions of problem F8 - Schwefel Function 2.26. The top graph describes the convergence from iteration 0 to iteration 100. The lower graph describes the moment in which the best aptitude is obtained during the execution of the algorithm. 

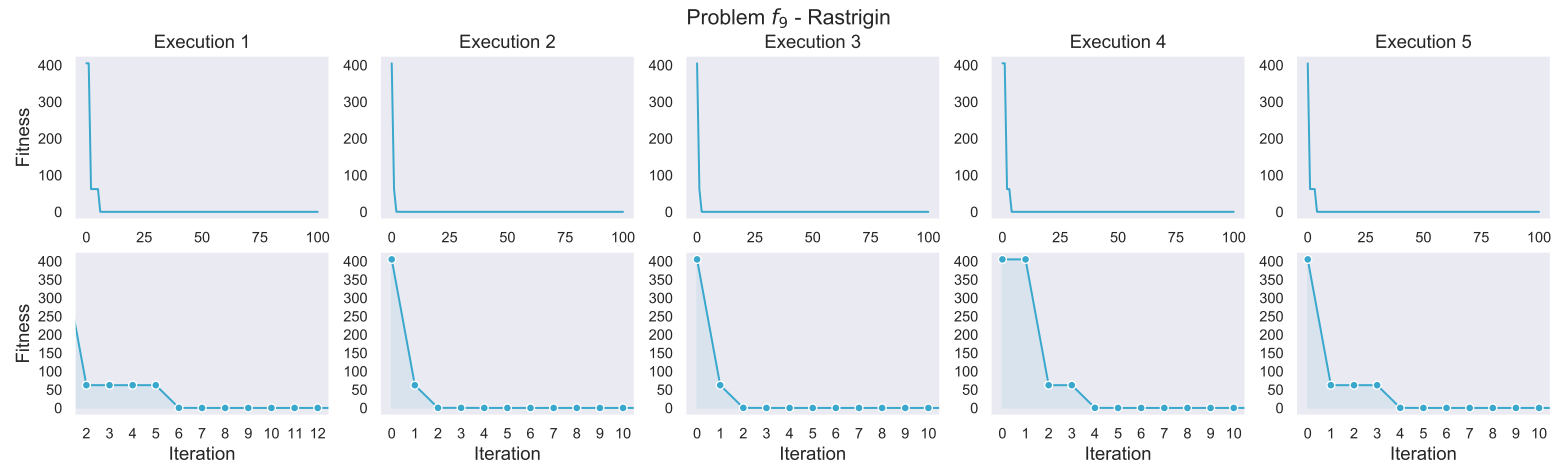

Figure 13. Convergence graph for five executions of problem F9 - Rastrigin. The top graph describes the convergence from iteration 0 to iteration 100 . The lower graph describes the moment in which the best aptitude is obtained during the execution of the algorithm.
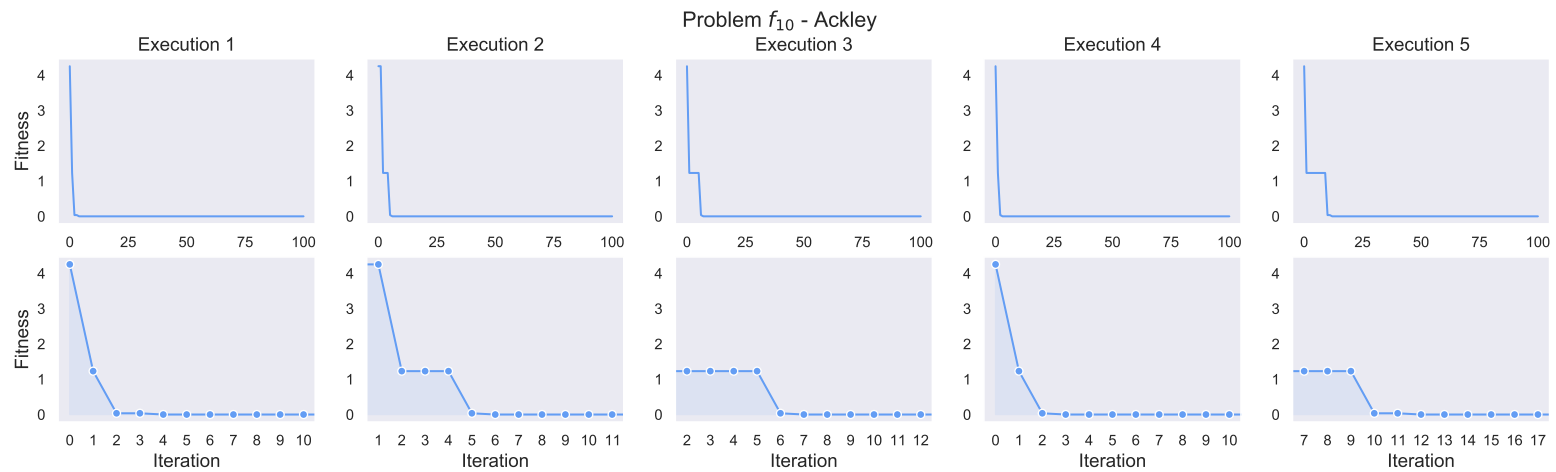

Figure 14. Convergence graph for five executions of problem F10 - Ackley. The top graph describes the convergence from iteration 0 to iteration 100 . The lower graph describes the moment in which the best aptitude is obtained during the execution of the algorithm.
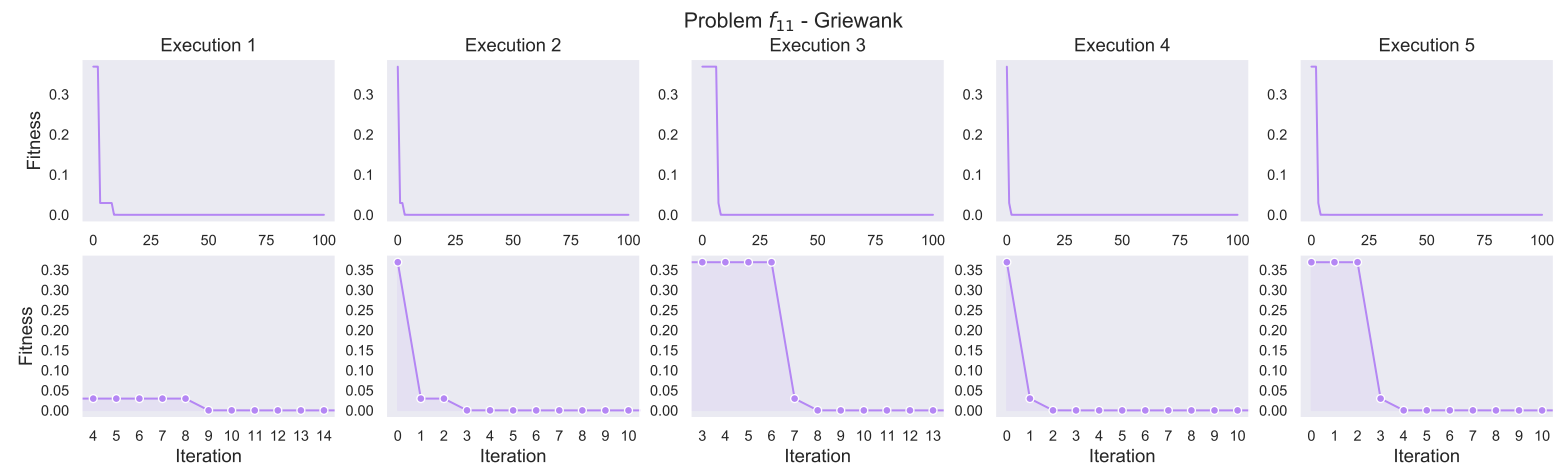

Figure 15. Convergence graph for five executions of problem F11 - Griewank. The top graph describes the convergence from iteration 0 to iteration 100 . The lower graph describes the moment in which the best aptitude is obtained during the execution of the algorithm. 

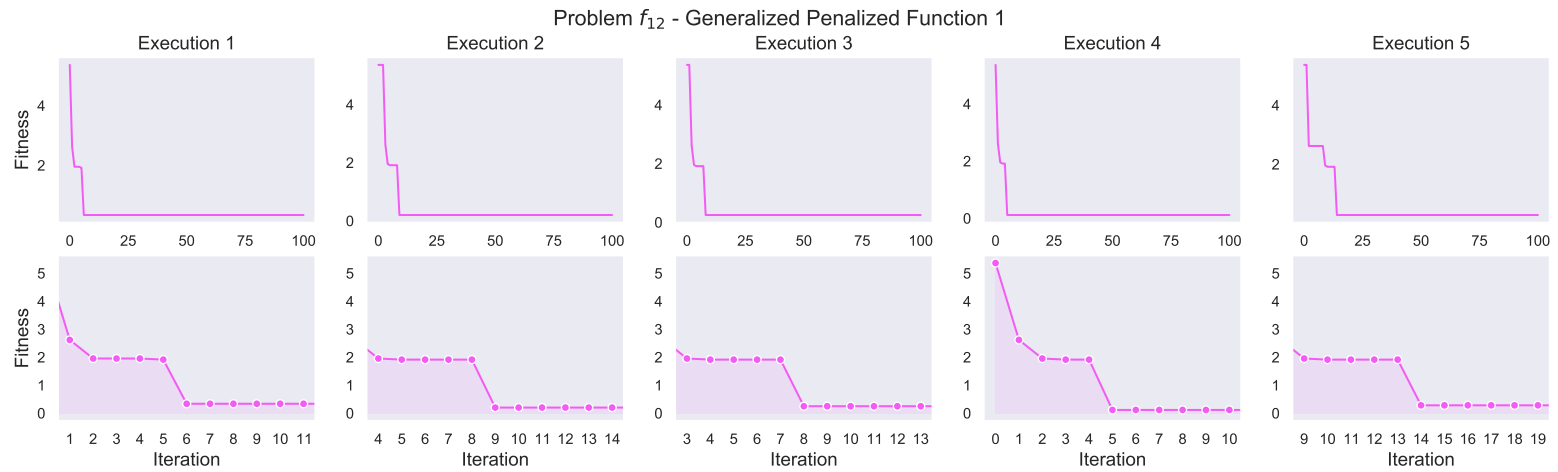

Figure 16. Convergence graph for five executions of problem F12 - Generalized Penalized Function 1. The top graph describes the convergence from iteration 0 to iteration 100. The lower graph describes the moment in which the best aptitude is obtained during the execution of the algorithm.

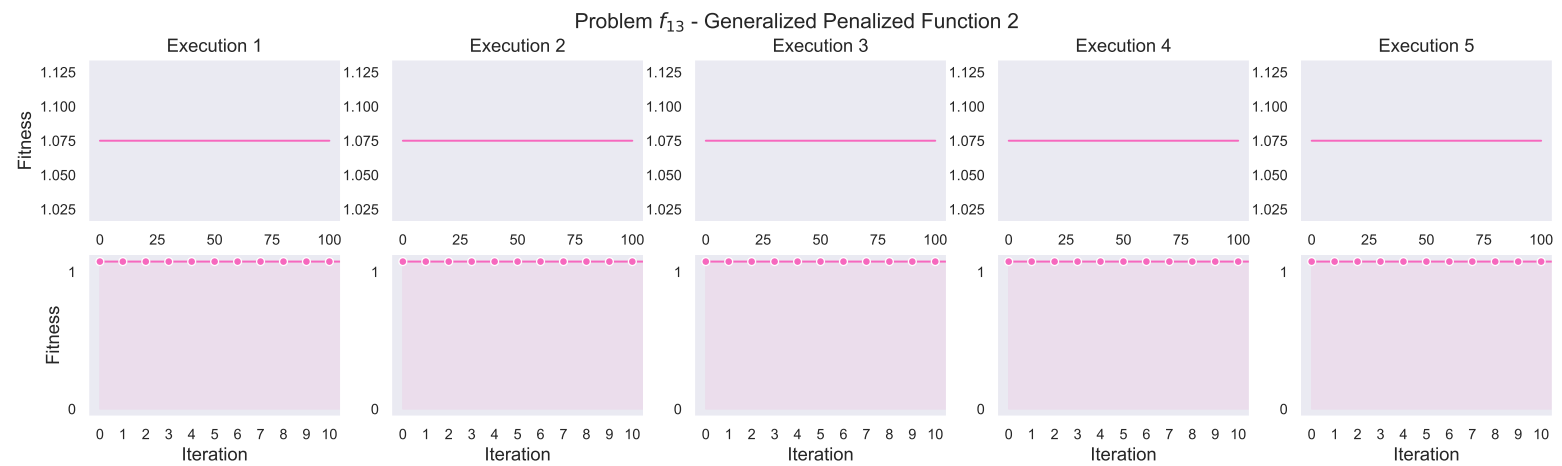

Figure 17. Convergence graph for five executions of problem F13 - Generalized Penalized Function 2. The top graph describes the convergence from iteration 0 to iteration 100. The lower graph describes the moment in which the best aptitude is obtained during the execution of the algorithm. 


\section{Discussion}

According to the results of table 7 , the best algorithm that has been found through the evolutionary process was able to find the value of the global optimum of 9 out of 13 optimisation problems. The problems are P1, P2, P3, P4, P6, P7, P9, P10, and P11, where the best fitness found with the value 0.0000, with a standard deviation and average fitness value of 0.0000 . Considering the convergence graphs 5 , $6,7,8,10,11,13$, and 14 , it can be seen that the optimal global value was reached during the first 10 of the 100 iterations in which the algorithm was executed. These data provide visual evidence that the algorithm managed to find the optimal global value with a limited number of iterations.

For problem P12, the best fitness was obtained for a value of 0.1167 , with a standard deviation of 0.0749 and an average value of 0.2373 . Problems P5, P8, and P13 did not achieve good fitness values; their standard deviation is 0.0000 . When performing a visual inspection on convergence graphs 9,6 , and 7 , it can be observed that from a point in their execution, the algorithms cannot obtain a new fitness value, maintaining a single value throughout the iteration. This may be because the instructions that make up the proposed algorithm are not suitable for those specific problems. This point is important because we can obtain important information that this group of problems can be solved with another technique or generate tests with only those optimisation problems.

Considering the results of section 3 of the instructions obtained from the best algorithm found. These instructions can be translated into pseudocode, so that a final display algorithm found can be seen in Figure 18.

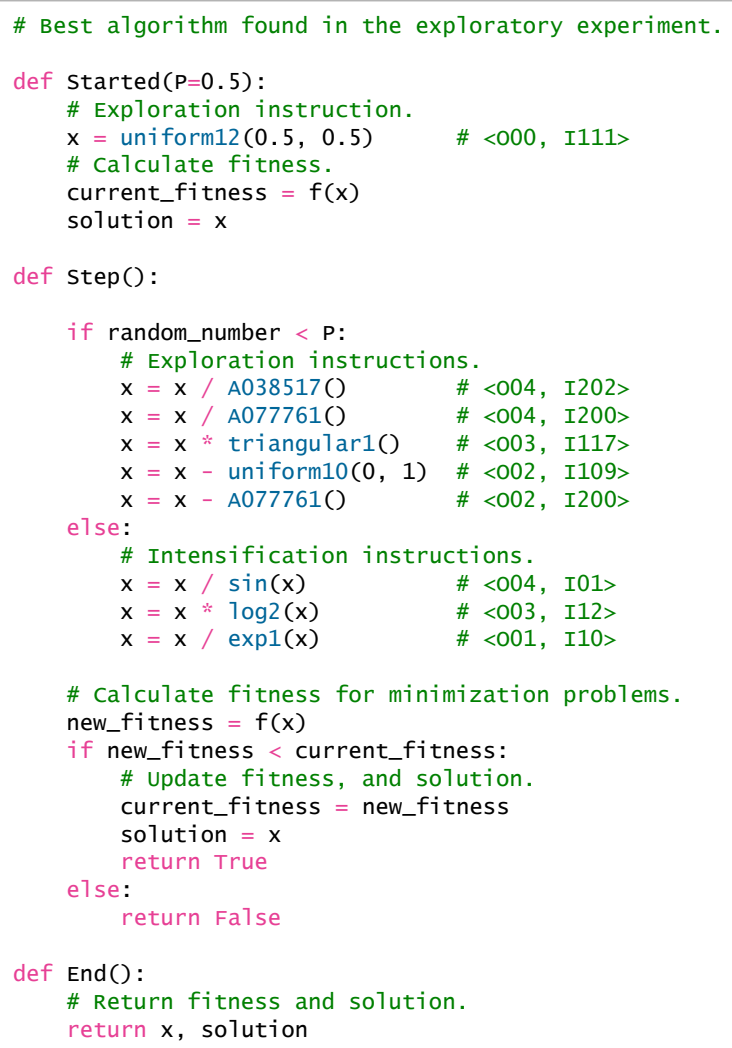

Figure 18. Best algorithm found during the 200 evolutionary iterations.

Finally, this work introduces a reinforced learning framework to generate metaheuristic algorithms (AutoMH). According to the exploratory tests carried out, the AutoMH has managed to find a metaheuristic algorithm that finds the optimal global value of 9 of the 13 continuous optimisation 
problems. New operators, or new indivisible intensification and exploration functions, can be integrated as future work, in such a way that the variety of new metaheuristic algorithms that can be found is enriched. Another point is to use a greater number of non-intelligent agents (the experiment was carried out with 30 agents), to increase the options of having a greater number of proposed algorithms that solve the problems entered.

Author Contributions: Conceptualisation, B.A.; methodology, B.A.; software, B.A.; validation, B.A.; formal analysis, B.A.; investigation, B.A.; resources, B.A.; data curation, B.A.; writing-original draft preparation, B.A.; writing-review and editing, B.A.; visualisation, B.A.; supervision, B.A.; project administration, B.A.; funding acquisition, B.A. All authors have read and agreed to the published version of the manuscript.

Funding: This research was privately funded by PhD (h.c.) Sonia Álvarez. This research does not receive external funding from governments, universities or companies.

Conflicts of Interest: The authors declare no conflict of interest. 


\section{Appendix A}

\section{Appendix A.1}

The appendix contains details of the continuous optimisation dataset for P01 to P13 functions.

Definition A1. P01 - Sphere. The Sphere function is defined by the objective function A1. The function is defined and evaluated in the input domain $x_{i} \in[-100,100]$ for all $i=\{1,2, \ldots, d\}$. The function has one global minimum at $f_{\min }\left(x^{*}\right)=0$ with $x^{*}=[0,0, \ldots, 0]$.

$$
f(x)=\sum_{i=1}^{d} x_{i}^{2}
$$

Definition A2. P02 - Schwefel 2.22. The Schwefel 2.22 function is defined by the objective function A2. The function is defined and evaluated in the input domain $x_{i} \in[-10,10]$ for all $i=\{1,2, \ldots, d\}$. The function has one global minimum at $f_{\min }\left(x^{*}\right)=0$ with $x^{*}=[0,0, \ldots, 0]$.

$$
f(x)=\sum_{i=1}^{d}\left|x_{i}\right|+\prod_{i=1}^{d}\left|x_{i}\right|
$$

Definition A3. P03 - Schwefel 1.2. The Schwefel 1.2 function is defined by the objective function A3. The function is defined and evaluated in the input domain $x_{i} \in[-100,100]$ for all $i=\{1,2, \ldots, d\}$. The function has one global minimum at $f_{\min }\left(x^{*}\right)=0$ with $x^{*}=[0,0, \ldots, 0]$.

$$
f(x)=\sum_{i=1}^{d}\left(\sum_{j=1}^{i} x_{j}\right)^{2}
$$

Definition A4. P04-Schwefel 2.21. The Schwefel 2.21 function is defined by the objective function A4. The function is defined and evaluated in the input domain $x_{i} \in[-100,100]$ for all $i=\{1,2, \ldots, d\}$. The function has one global minimum at $f_{\min }\left(x^{*}\right)=0$ with $x^{*}=[0,0, \ldots, 0]$.

$$
f(x)=\max _{i=1, \ldots, d}\left|x_{i}\right|
$$

Definition A5. P05-Rosenbrock's. The Rosenbrock's function is defined by the objective function A5. The function is defined and evaluated in the input domain $x_{i} \in[-30,30]$ for all $i=\{1,2, \ldots, d\}$. The function has one global minimum at $f_{\min }\left(x^{*}\right)=0$ with $x^{*}=[1,1, \ldots, 1]$.

$$
f(x)=\sum_{i=1}^{d-1}\left[100\left(x_{i+1}-x_{i}^{2}\right)^{2}+\left(1-x_{i}\right)^{2}\right]
$$

Definition A6. P06 - Step. The Step function is defined by the objective function A6. The function is defined and evaluated in the input domain $x_{i} \in[-100,100]$ for all $i=\{1,2, \ldots, d\}$. The function has one global minimum at $f_{\min }\left(x^{*}\right)=0$ with $x^{*}=\left[x_{1}, x_{2}, \ldots, x_{d}\right], x_{i} \in[-0.5,0.5)$.

$$
f(x)=\sum_{i=1}^{d}\left(\left\lfloor x_{i}+0.5\right\rfloor\right)^{2}
$$

Definition A7. P07 - Noisy Quartic. The Noisy Quartic function is defined by the objective function A7. The function is defined and evaluated in the input domain $x_{i} \in[-1.28,1.28]$ for all $i=\{1,2, \ldots, d\}$. The function 
has one global minimum at $f_{\min }\left(x^{*}\right)=0+\sum_{i=1}^{d} \eta_{i}$ with $x^{*}=[0,0, \ldots, 0]$. Where, $\eta$ is a random number bounded between $[0,1)$.

$$
f(x)=\sum_{i=1}^{d}\left(i x_{i}^{4}+\eta_{i}\right)
$$

Definition A8. P08 - Schwefel Function 2.26. The Schwefel function 2.26 is defined by the objective function A8. The function is defined and evaluated in the input domain $x_{i} \in[-500,500]$ for all $i=$ $\{1,2, \ldots, d\}$. The function has one global minimum at $f_{\min }\left(x^{*}\right)=0$ with $x^{*}=[4.209687462275036 e+$ $002,4.209687462275036 e+002, \ldots, 4.209687462275036 e+002]$.

$$
f(x)=4.189828872724338 e+002 \times d-\sum_{i=1}^{d} x_{i} \sin \left(\sqrt{\left|x_{i}\right|}\right)
$$

Definition A9. P09-Rastrigin Function. The Rastrigin function 2.26 is defined by the objective function A9. The function is defined and evaluated in the input domain $x_{i} \in[-5.12,5.12]$ for all $i=\{1,2, \ldots, d\}$. The function has one global minimum at $f_{\min }\left(x^{*}\right)=0$ with $x^{*}=[0,0, \ldots, 0]$.

$$
f(x)=\sum_{i=1}^{d}\left[x_{i}^{2}-10 \cos \left(2 \pi x_{i}\right)+10\right]
$$

Definition A10. P10 - Ackley Function. The Ackley function is defined by the objective function A10. The function is defined and evaluated in the input domain $x_{i} \in[-32,32]$ for all $i=\{1,2, \ldots, d\}$. The function has one global minimum at $f_{\min }\left(x^{*}\right)=0$ with $x^{*}=[0,0, \ldots, 0]$.

$$
f(x)=-a \exp \left(-b \sqrt{\frac{1}{d} \sum_{i=1}^{d} x_{i}^{2}}\right)-\exp \left(\frac{1}{d} \sum_{i=1}^{d} \cos \left(c x_{i}\right)\right)+a+\exp (1)
$$

where : $a=20$

$$
b=0.2
$$

Definition A11. P11 - Griewank function. The Griewank function is defined by the objective function A11. The function is defined and evaluated in the input domain $x_{i} \in[-600,600]$ for all $i=\{1,2, \ldots, d\}$. The function has one global minimum at $f_{\min }\left(x^{*}\right)=0$ with $x^{*}=[0,0, \ldots, 0]$.

$$
f(x)=\frac{1}{4000} \sum_{i=1}^{d} x_{i}^{2}-\prod_{i=1}^{d} \cos \left(\frac{x_{i}}{\sqrt{i}}\right)+1
$$


Definition A12. P12 - Generalized Penalized Function 1. The Generalized Penalized function 1 is defined by the objective function A12. The function is defined and evaluated in the input domain $x_{i} \in[-50,50]$ for all $i=\{1,2, \ldots, d\}$. The function has one global minimum at $f_{\min }\left(x^{*}\right)=0$ with $x^{*}=[-1,-1, \ldots,-1]$.

$$
\begin{aligned}
f(x) & =\frac{\pi}{d} \times\left\{10 \sin ^{2}\left(\pi y_{1}\right)+\sum_{i=1}^{d-1}\left[\left(y_{i}-1\right)^{2}\left(1+10 \sin ^{2}\left(\pi y_{i+1}\right)\right)\right]+\left(y_{d}-1\right)^{2}\right\}+\sum_{i=1}^{d} u\left(x_{i}, a, k, m\right) \\
y_{i} & =1+\frac{1}{4}\left(x_{i}+1\right) \quad u\left(x_{i}, a, k, m\right)= \begin{cases}k\left(x_{i}-a\right)^{m} & \text { if } x_{i}>a \\
0 & \text { if }-a \leq x_{i} \leq a \\
k\left(-x_{i}-a\right)^{m} & \text { if } x_{i}<-a\end{cases} \\
a & =10 \\
k & =100 \\
m & =4
\end{aligned}
$$

Definition A13. P13 - Generalized Penalized Function 2. The Generalized Penalized function 2 is defined by the objective function A13. The function is defined and evaluated in the input domain $x_{i} \in[-50,50]$ for all $i=\{1,2, \ldots, d\}$. The function has one global minimum at $f_{\min }\left(x^{*}\right)=0$ with $x^{*}=[1,1, \ldots, 1]$.

$$
\begin{aligned}
f(x)= & 0.1 \times\left\{\sin ^{2}\left(3 \pi x_{1}\right)+\sum_{i=1}^{d-1}\left[\left(x_{i}-1\right)^{2}\left(1+\sin ^{2}\left(3 \pi x_{i+1}\right)\right)\right]+\left[\left(x_{n}-1\right)^{2}\left(1+\sin ^{2}\left(2 \pi x_{n}\right)\right)\right]\right\}+ \\
& \sum_{i=1}^{d} u\left(x_{i}, a, k, m\right) \\
y_{i}= & 1+\frac{1}{4}\left(x_{i}+1\right) \quad u\left(x_{i}, a, k, m\right)= \begin{cases}k\left(x_{i}-a\right)^{m} & \text { if } x_{i}>a \\
0 & \text { if }-a \leq x_{i} \leq a \\
k\left(-x_{i}-a\right)^{m} & \text { if } x_{i}<-a\end{cases} \\
a= & 5 \\
k= & 100 \\
m= & 4
\end{aligned}
$$




\section{References}

1. Tovey, C.A. Nature-Inspired Heuristics: Overview and Critique. In Recent Advances in Optimization and Modeling of Contemporary Problems; INFORMS, 2018; pp. 158-192.

2. Kaelbling, L.P.; Littman, M.L.; Moore, A.W. Reinforcement learning: A survey. Journal of artificial intelligence research 1996, 4, 237-285.

3. Hein, D.; Hentschel, A.; Runkler, T.A.; Udluft, S. Particle swarm optimization for model predictive control in reinforcement learning environments. In Critical Developments and Applications of Swarm Intelligence; IGI Global, 2018; pp. 401-427.

4. Nazari, M.; Oroojlooy, A.; Snyder, L.; Takác, M. Reinforcement learning for solving the vehicle routing problem. Advances in Neural Information Processing Systems 2018, 31, 9839-9849.

5. Sadeg, S.; Hamdad, L.; Remache, A.R.; Karech, M.N.; Benatchba, K.; Habbas, Z. QBSO-FS: A Reinforcement Learning Based Bee Swarm Optimization Metaheuristic for Feature Selection. International Work-Conference on Artificial Neural Networks. Springer, 2019, pp. 785-796.

6. Hayashi, K.; Ohsaki, M. Reinforcement learning for optimum design of a plane frame under static loads. Engineering with Computers 2020, pp. 1-13.

7. Solozabal, R.; Ceberio, J.; Takáč, M. Constrained combinatorial optimization with reinforcement learning. arXiv preprint arXiv:2006.11984 2020.

8. Calvet, L.; de Armas, J.; Masip, D.; Juan, A.A. Learnheuristics: hybridizing metaheuristics with machine learning for optimization with dynamic inputs. Open Mathematics 2017, 15, 261-280.

9. Barrett, T.; Clements, W.; Foerster, J.; Lvovsky, A. Exploratory combinatorial optimization with reinforcement learning. Proceedings of the AAAI Conference on Artificial Intelligence, 2020, Vol. 34, pp. 3243-3250.

10. Kanda, J.; de Carvalho, A.; Hruschka, E.; Soares, C.; Brazdil, P. Meta-learning to select the best meta-heuristic for the traveling salesman problem: A comparison of meta-features. Neurocomputing 2016, 205, 393-406.

11. Yu, S.; Aleti, A.; Barca, J.C.; Song, A. Hyper-heuristic online learning for self-assembling swarm robots. International Conference on Computational Science. Springer, 2018, pp. 167-180.

12. de Santiago Júnior, V.A.; Özcan, E.; de Carvalho, V.R. Hyper-Heuristics based on Reinforcement Learning, Balanced Heuristic Selection and Group Decision Acceptance. Applied Soft Computing 2020, 97, 106760.

13. Wai, H.T.; Yang, Z.; Wang, Z.; Hong, M. Multi-agent reinforcement learning via double averaging primal-dual optimization. Advances in Neural Information Processing Systems 2018, 31, 9649-9660.

14. Cadenas, J.M.; Garrido, M.C.; Muñoz, E. Using machine learning in a cooperative hybrid parallel strategy of metaheuristics. Information Sciences 2009, 179, 3255-3267.

15. Real, E.; Liang, C.; So, D.; Le, Q. AutoML-zero: evolving machine learning algorithms from scratch. International Conference on Machine Learning. PMLR, 2020, pp. 8007-8019.

16. Talbi, E.G. Combining metaheuristics with mathematical programming, constraint programming and machine learning. Annals of Operations Research 2016, 240, 171-215.

17. Talbi, E.G. Machine learning into metaheuristics: A survey and taxonomy of data-driven metaheuristics 2020.

18. Bengio, Y.; Lodi, A.; Prouvost, A. Machine learning for combinatorial optimization: a methodological tour d'horizon. European Journal of Operational Research 2020. 